ses Ergebnis war überraschend, weil $4 \mathrm{~Pa}$ tienten den prognostisch ungünstigen Subtyp des multifokalen diffus-großzelligen B-Zell-Lymphoms vom Leg-Type aufwiesen. Die Dosis war mit $20 \mathrm{mg} / \mathrm{m}^{2}$ niedrig gewählt. In Folgestudien soll dieses nebenwirkungsarme und einfach zu applizierende Medikament an einer höheren Fallzahl weiter untersucht werden [37].

\section{Fazit für die Praxis}

- Die Diagnose „primär kutanes B-ZellLymphom" (PKBZL) beruht in erster Linie auf dem Ergebnis histologischer Untersuchungen und einer Staginguntersuchung. Entscheidend für die histologische Beurteilung ist eine einwandfreie technische Qualität des Präparats.

- Für das exakte Staging sind neben konventionellen Laboruntersuchungen (einschließlich Immunelektrophorese), Abdomensonographie (Leber, Milzgröße, intraperitoneale Lymphome) und Thoraxröntgenuntersuchung bei entsprechender klinischer Symptomatik weitere Spezialuntersuchungen (z. B. Beckenkammbiopsie, Gastroskopie) und bildgebende radiologische Verfahren einschließlich PET angezeigt.

- Therapeutisch ist zu beachten, dass der größte Teil der PKBZL den indolenten Formen angehört und somit nicht primär mit einer Chemotherapie zu behandeln ist.

- In der Regel wird Rituximab als Monotherapie eingesetzt, um ein „Übertherapieren" indolenter Lymphome zu vermeiden.

-Weitere therapeutische Möglichkeiten sind Strahlentherapie und neuere Ansätze mit Elektrochemotherapie sowie pegyliertem Doxorubicin.

\section{Korrespondenzadresse:}

Priv.-Doz. Dr. med. H.-J. Schulze

Dermatologie der Fachklinik Hornheide Leiter des Hauttumorzentrum Hornheide Münster, Dorbaumstr. 300

48157 Münster

schulze@fachklinik-hornheide.de

\title{
Mantelzell-Lymphom: Neue Therapie verdoppelt krankheitsfreie Zeit
}

\author{
Die meisten Patienten, die an einem \\ Mantelzell-Lymphom erkranken, sind \\ älter als $\mathbf{6 0}$ Jahre. Aufgrund ihres oft \\ schlechten Gesundheitszustandes \\ können sie sich keiner hochaggressiven \\ Therapie unterziehen. Aktuellen \\ Leitlinien zufolge erhalten sie deshalb \\ eine mit dem Antikörper Rituximab \\ kombinierte Chemotherapie, mit der \\ das Mantelzell-Lymphom für eine \\ begrenzte, oft nur kurze Dauer \\ zurückgedrängt werden kann.
}

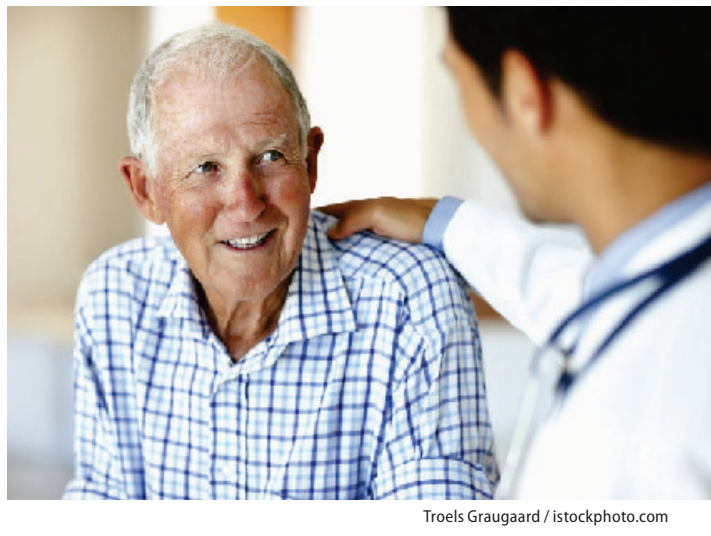

Mittels einer Erhaltungstherapie versuchen Ärzte, die krankheitsfreie Zeit bis zum Wiederauftreten des Mantelzell-Lymphoms auszudehnen. Das bislang verwendete Interferon-alpha weist jedoch starke Nebenwirkungen auf. Rituximab ist besser verträglich und kann zudem die krankheitsfreie Zeit von MCL-Patienten erheblich verlängern. Die erste Zwischenauswertung der MCL-Elderly-Studie des Europäischen Mantelzell-Lymphom Netzwerks (EMCLN) belegt eindrucksvoll, dass bei älteren Patienten eine vormals aggressive Erkrankung in einen eher chronischen Verlauf überführt werden kann.

\section{Krankheitsfreie Zeit: 51 Monate gegenüber 24 Monaten}

Über 500 Patienten aus acht europäischen Ländern erhielten als Primärtherapie nach dem Zufallsprinzip entweder acht Zyklen R-CHOP (Rituximab, Cyclophosphamid, Doxorubin, Vincristin und Prednisolon) oder 6 Zyklen R-FC (Rituximab, Fludarabin und Cyclophosphamid). Patienten, deren Mantelzell-Lymphom durch diese Therapien ganz oder teilweise zurückgedrängt wurde, erhielten anschließend, bis die Krankheit wieder aktiv wurde, entweder eine Rituximab-oder eine Interferon-alpha-Erhaltungstherapie.

Die krankheitsfreie Zeit der Patienten mit Rituximab-Erhaltungstherapie betrug 51 Monate, gegenüber 24 Monaten der mit Interferon-alpha behandelten Patienten. Nach drei Jahren betrug das Gesamtüberleben der Patienten mit R-CHOP-Behandlung gar 85\% nach Rituximab, gegenüber $70 \%$ nach Interferon.

\section{Nebenwirkungen beeinflussen die Therapiebereitschaft}

Erwartungsgemäß waren die Nebenwirkungen von Rituximab geringer als die von Interferon: Eine Schädigung der Blutzellen trat unter Interferon doppelt so häufig auf wie unter Rituximab. 61\% der mit Interferon behandelten Patienten brach die Erhaltungstherapie vorzeitig - also bevor erneut Krankheitszeichen aufgetreten sind - ab. In der Gruppe der mit Rituximab behandelten Patienten betrug dieser Anteil nur 30\%. Dass die Erhaltungstherapie jedoch einen großen Einfluss auf die krankheitsfreie Zeit und das Gesamtüberleben hat verdeutlichen die Krankheitsverläufe jener Patienten, die aus unterschiedlichen Gründen keine Erhaltungstherapie erhalten hatten. Ihre krankheitsfreie Zeit betrug nur 26 Monate. Das Gesamtüberleben lag nach drei Jahren nur bei 52\%.

Obwohl Rituximab gegenwärtig noch nicht für die Erhaltungstherapie beim MantelzellLymphom zugelassen ist, stellt diese Erhaltungstherapie im Anschluss an eine R-CHOP Induktionstherapie einen neuen, gut verträglichen Therapiestandard für ältere MCL-Patienten dar. 